from sphere to spheroid, with $\mu \neq 2$, yields a structurally stable caustic.

To establish directly that the umbilics calculated above are indeed hyperbolic rather than elliptic would have required a complicated analysis of the cubic terms in the expression for the wavefront as it was refracted and reflected astigmatically through the spheroidal water drop. But recognizing that the umbilics are part of the unfolding of a higher catastrophe removes the need for this calculation, for there is no reason to suspect that they change their character when the unfolding is finite rather than infinitesimal, as assumed in the theory. Note, however, that I have calculated the critical $D / H$ ratios exactly, rather than merely to lowest order in $2-\mu$ and $D / H-1$.

I thank Drs Marston and Trinh for showing me their letter before publication.

Received 14 September; accepted 6 November 1984.

1. Marston, P. L. \& Trinh, E. H. Nature 312, 529-531 (1984).

2. Berry, M. V. \& Upstill, C. Prog. Opt. 18, 257-345 (1980).

\section{A very narrow Mössbauer resonance in ${ }^{63} \mathrm{Ni}$}

\section{H. Muthig, E. Huenges \& H. Morinaga}

Physics Department, Technical University Munich, 8046 Garching, FRG

Investigations of the Mössbauer effect in nickel have been less fruitful than those on iron, because until now there has been no convenient resonance akin to the 14-keV transition (half-life $98 \mathrm{~ns}$ ) in ${ }^{57} \mathrm{Fe}$. So far the only available Mössbauer resonance in nickel has been the 67-keV transition (half-life, $5 \mathrm{~ns}$ ) in ${ }^{61} \mathrm{Ni}$, whose natural resonance line is so broad that its width is comparable to the nuclear Zeeman splitting in ferromagnetic nickel metal. The result is a spectrum comprising a single broad line instead of a well-resolved splitting into discrete lines, as is the case for narrow resonances. We have recently succeeded in a scattering experiment in observing the Mössbauer effect in the $87-\mathrm{keV}$ transition (halflife $1.7 \mu \mathrm{s})$ in radioactive ${ }^{63} \mathrm{Ni}\left(t_{1 / 2}=100 \mathrm{yr}\right)$. Because of the long lifetime of this state, its natural linewidth is smaller than that of ${ }^{61} \mathrm{Ni}(\times 330)$, and the commonly used ${ }^{57} \mathrm{Fe}(\times 17)$, but still larger than that of ${ }^{67} \mathrm{Zn}(\times 0.2)$, which has the highest relative energy resolution of all Mössbauer resonances in use. The use of ${ }^{63} \mathrm{Ni}$ should thus permit us in future to perform detailed Mössbauer studies in nickel.

The $\gamma$-source for observing the Mössbauer effect in ${ }^{63} \mathrm{Ni}$ is ${ }^{63} \mathrm{Co}$ (half-life 27.5 -s), produced by the ${ }^{64} \mathrm{Ni}(t, \alpha){ }^{63}$ Co reaction with the triton beam of the Technical University of Munich Compact Cyclotron ${ }^{1}$. With this reaction, ${ }^{63} \mathrm{Co}$ could be produced essentially free of unwanted radioactivity, which is not the case when using other projectiles. A production rate for ${ }^{63} \mathrm{Co}$ of $\sim 1 \mathrm{mCi} \mathrm{min}^{-1}$ was possible during irradiation with a triton beam of $\sim 2 \mu \mathrm{A}$.

A total of 15 targets were used to avoid accumulation of long-lived activity by repeated irradiation. The target material was metallic nickel foil of $27 \mathrm{mg} \mathrm{cm}^{-2}$ thickness and $10 \mathrm{~mm}$ diameter, made of enriched $(97.7 \%){ }^{64} \mathrm{Ni}$. These foils were soldered to coin-shaped target holders made of copper disks $6 \mathrm{~mm}$ thick and $25 \mathrm{~mm}$ in diameter. A hole of $10-\mathrm{mm}$ diameter was bored along the symmetry axis of the discs, leaving a wall-thickness of $0.5 \mathrm{~mm}$ at the bottom. The ${ }^{64} \mathrm{Ni}$ foils were soldered using lead solder into this cylindrical cavity under vacuum conditions. Before irradiation the targets were cooled to liquid nitrogen temperature. Because of the energy loss of the tritons in the target foil, we are sure that the temperature of the ${ }^{64} \mathrm{Ni}$ is higher than $77 \mathrm{~K}$ during irradiation presumably allowing annealing of radiation damage in the nickel lattice to occur. After irradiation, the heat dissipated in the nickel foil is absorbed owing to the high heat capacity of copper target holder, thus rapidly cooling the target foil again to the temperature of liquid nitrogen. The sources were then quickly transported, within $6-8 \mathrm{~s}$, to a measuring position, well-shielded from the accelerator. This was accomplished by rolling the targets through inclined tubes of rectangular cross-section. The absorber and scatterer was a ${ }^{63} \mathrm{Ni}$ foil $\left(12 \%{ }^{63} \mathrm{Ni}, 15-\mathrm{mm}\right.$ diameter, $0.2-\mathrm{mm}$ thickness), which was also cooled to liquid nitrogen temperature.

We have used $41 \mathrm{mg}$ of ${ }^{63} \mathrm{Ni}$ corresponding to $2.3 \mathrm{Ci}$ of the 100 -yr half-life activity. Ferromagnetic nickel metal was chosen as the chemical form of source and absorber (scatterer), and both are thus subject to a magnetic nuclear Zeeman splitting. Only the unshifted central resonance line was covered by the velocity drive. The relative Doppler velocity between the source and the absorber (scatterer) was obtained by moving the absorber sinusoidally with a piezo-drive. The maximum velocity was $35 \mu \mathrm{m} \mathrm{s}^{-1}$ and the velocity drive was calibrated by observing the well-known isomer shift between ${ }^{67} \mathrm{GaCu}$ and ${ }^{67} \mathrm{Zn}$ - $\beta$-brass. The scattered and transmitted radiation of the sources were measured simultaneously by two planar intrinsic germanium detectors and recorded by two multichannel-analysers synchronously with the velocity of the drive.

After more than 200 measuring cycles (altogether $10 \mathrm{~h}$ of measuring time) a resonance line was seen in the scattering spectrum with a linewidth of 5 times the minimum resonance width, having taken the scatterer thickness into account. This spectrum is shown in Fig. 1. Its intensity corresponded very well

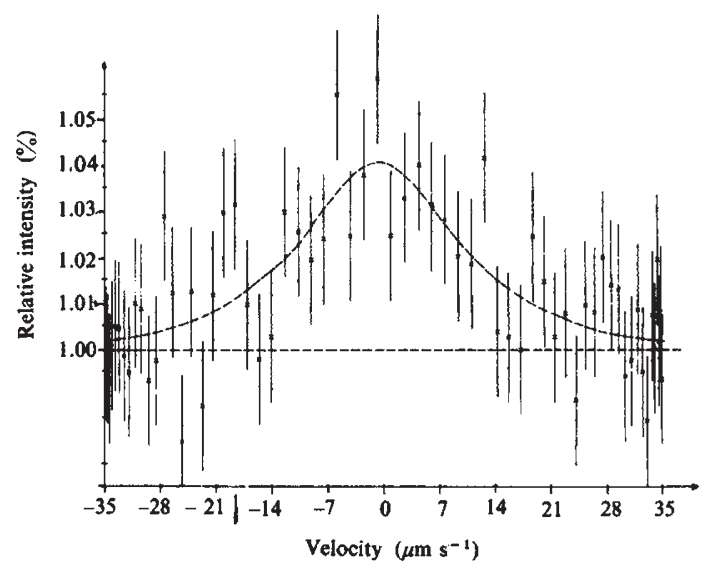

Fig. 1 Intensity of the recoil-less scattered radiation from a ${ }^{63} \mathrm{Ni}$ scatterer. (Only the central resonance line is observed.)

with the value calculated by applying to the data the procedure of Debrunner and Morrison ${ }^{2}$. This indicates that the adopted Debey-Waller factor for liquid nitrogen temperature, derived from ${ }^{61} \mathrm{Ni}$ experiments, reproduces the actual intensity very well. The resonance line disappeared when the temperature of source and scatterer was raised to room temperature. However, the statistics were still not good enough to observe the resonance in transmission geometry. The broadening of the line is most probably due to radiation damage in the source material.

We shall now try to reduce the linewidth and find a compound that gives an unsplit single emission line without generating too much unwanted radioactivity. Also certain basic experiments, such as the determination of the ground state magnetic moment of ${ }^{63} \mathrm{Ni}$, are being planned.

We thank the Deutsche Forschungsgemeinschaft for their support.

Received 23 August; accepted 11 October 1984.

1. Wegmann, H., Huenges, E., Muthig, H. \& Morinaga, H. Nuch Instrum. Meth. 179, 217-222 (1981).

2. Debrunner, P. \& Morrison, R. J. Rev. scient. Instrum. 36, 145-149 (1965). 